\title{
EXISTENCE IN THE LARGE OF TOTALLY GEODESIC SUBMANIFOLDS OF RIEMANNIAN SPACES
}

\author{
BY ROBERT HERMANN ${ }^{1}$ \\ Communicated by Hans Samelson, November 20, 1959
}

1. Let $M$ be a complete, $C^{\infty}$ Riemannian manifold, $x_{0}$ a point of $M$, and $N$ a subspace of $M_{x_{0}}$, the tangent space to $M$ at $x_{0}$. In this note we give necessary and sufficient conditions that there be a complete, immersed, $C^{\infty}$, totally geodesic subnanifold of $M$ through $x_{0}$, whose tangent space at $x_{0}$ is precisely $N$.

A once-broken geodesic starting from $x_{0}$ is said to be admissible if

(a) the tangent vector at any point of the geodesic belongs to $N$ when parallel-translated back along the geodesic to $x_{0}$

(b) the second piece of the geodesic lies in a geodesic-convex neighborhood of the end-point of the first piece.

For $x \in M, v_{1} \& v_{2} \in M_{x}$, let $R_{x}\left(v_{1}, v_{2}\right)$ be the skew-symmetric linear transformatiou of $M_{x}$ induced by the Riemann curvature tensor. For each admissible, once-broken geodesic $g$ let $N_{g} \subset M_{\text {end } g}$ be the subspace obtained by parallel-translating $N$ along $g$ to its end-point.

With these notations, we can state the result:

Theorem. Suppose that $R_{\text {end }}\left(N_{g}, N_{g}\right)\left(N_{g}\right) \subset N_{g}$ for each admissible, once-broken geodesic $g$. Then, there is an immersed, $C^{\infty}$, complete submanifold $X$ of $M$ whose tangent space at $x_{0}$ is $N$.

The local version of this theorem was (more or less) given by Cartan [2]. Our job is to extend this globally by some sort of "analytic continuation." The proof, which is only sketched here, is inspired by the proof of Ambrose's isometry theorem [1]. One should notice then that conversely Ambrose's theorem is a consequence. Given two Riemannian manifolds $M^{\prime}$ and $M^{\prime \prime}$ that one hopes to show isometric, one looks for the graph of the isometry as a totally geodesic submanifold of $M=M^{\prime} \times M^{\prime \prime}$.

I wish to thank H. Samelson for valuable suggestions.

2. The proof. First, we are going to define $X$ and a map $\phi: X \rightarrow M$ as a set of points, and then try to make $X$ into a $C^{\infty}$ manifold in such a way that $\phi$ is an immersion.

We need some local facts. Recall the definition of the map Exp: $M_{x} \rightarrow M$ for $x \in M$. If $v \in M_{x}, \operatorname{Exp} v$ is the end point of the

1 This research was supported in part by OOR, U. S. Army under contract number DA 19-020-ORD-3778. 
geodesic starting at $x$, which points in the direction of $v$, and whose length is the length of $v$.

Lemma 1.1. For fixed $x \in M$, let $U$ be an open set of $M_{x}$ such that (a) for $v \in U$, $t v \in U$ for $0 \leqq t \leqq 1$ and (b) Exp: $U \rightarrow M$ is a diffeomorphism of $U$ with an open neighborhood of $x$.

Let $N$ be a subspace of $M_{x}$. For $v \in N \cap U$, let $N_{v} \subset M_{\operatorname{Exp} v}$ be the subspace obtained by parallel translating $N$ along the unique geodesic in $\operatorname{Exp} U$ joining $x$ to $\operatorname{Exp} v$. Suppose that $R_{\operatorname{Exp} v}\left(N_{v}, N_{v}\right)\left(N_{v}\right) \subset N_{v}$.

Then, $\operatorname{Exp}(N \cap U)$ is a totally geodesic submanifold of $M$. In particular, its tangent space at each Exp $v$ is just $N_{v}$. For $v_{1}, v_{2} \in N \cap U$ the result of parallel-translating $N_{v}$, along any path joining $\operatorname{Exp} v_{1}$ to $\operatorname{Exp} v_{2}$ in $\operatorname{Exp}(N \cap U)$ is just $N_{v 2}$. Any sufficiently small geodesic in $\operatorname{Exp}(U)$ whose end-points lie on $\operatorname{Exp}(N \cap U)$ lies completely in $\operatorname{Exp}(N \cap U)$.

The proof is in [2].

Now, define $X$ as follows: A point $\mathrm{x}$ of $X$ is a pair $\left(x, N_{\mathbf{x}}\right)$ consisting of a point $x \in M$ such that $x=$ end-point of $g$ and $N_{\mathbf{x}}=N_{\theta}$ for at least one admissible, once-broken geodesic $g . \phi: X \rightarrow M$ is then defined by: $\phi(x)=x$.

For any sufficiently small open neighborhood $U$ of 0 in $N_{\mathbf{x}}$, define a subset $U$ of $X$ as follows:

$y=\left(y, N_{y}\right) \in U$ if and only if $y=\operatorname{Exp} v$ for some $v \in U$, and $N_{y}$ is the result of parallel-translating $N_{x}$ along the geodesic $t \rightarrow \operatorname{Exp} t v$ $(0 \leqq t \leqq 1)$, from $x$ to $y$.

(Notice that the condition (b) for admissible geodesics and the local properties of totally geodesic submanifolds guarantee that $\operatorname{Exp}(U) \subset \phi(X)$ if $U$ is sufficiently small.)

Define the topology on $X$ so that these sets $U$ are a basis for the open sets. One proves, using the local Lemma 1.1, that

(a) the topology is Hausdorff;

(b) the intersection $U \cap U^{\prime}$ of two such sets associated to $\left(x, N_{\mathbf{x}}\right)$ $\&\left(x^{\prime}, N_{x^{\prime}}\right) \in X$ correspond, under the identifications $U \leftrightarrow U \subset N_{x}$, $U^{\prime} \leftrightarrow U^{\prime} \subset N_{x^{\prime}}$, to open sets of $U$ and $U^{\prime}$.

Then, we can use the identification of $U$ with $U$, an open subset of Euclidean space, to make $X$ into a $C^{\infty}$ manifold. $\phi$ is then a $C^{\infty}$ immersion of $X$ in $M$, since $\phi: U \rightarrow M$ can be identified with Exp: $U \rightarrow M$.

Now, $\operatorname{Exp}(U)$ is an imbedded, totally geodesic submanifold of $M$. Hence, if we make $X$ into a Riemannian manifold in the obvious way, i.e. so $U$ is isometric with $\operatorname{Exp}(U)$, then $X$ is a totally geodesic sub- 
manifold of $M$ in the sense that $\phi$ maps geodesics of $X$ into geodesics of $M$. It is complete, since all geodesics starting from $\mathrm{x}_{0}=\left(x_{0}, N\right)$ can be indefinitely extended. The proof is then finished.

3. Further remarks. The theorem raises two general problems. First, what is the most general range of applicability in differential geometry of the method of "analytic continuation"? Second, how may global families of geodesics be put together to form nonsingular submanifolds?

If $M$ and the metric are real analytic, the hypothesis of the theorem can be given a strictly local form. To do this, introduce $\Delta^{n} R_{x_{0}}$, the $n$th covariant derivative of $R$ at $x_{0}$ : For $v_{1}, \cdots, v_{n+2} \in M_{x_{0}}$, $\Delta^{n} R_{x_{0}}\left(v_{1}, v_{2}: v_{3}, \cdots, v_{n+2}\right)$ is a skew-symmetric endomorphism of $M_{x_{0}}$. The condition we have in mind is then that it always maps $N$ into itself.

Following the pattern established by N. Hicks [3] in generalizing Ambrose's theorem, there seem to be no real difficulties in extending the constructions made above to the case of a complete affine connection, provided of course that one extends the class of admissible curves to include geodesics with an arbitrary number of components. It is not clear however whether one can prove that the resulting manifold is complete, since a powerful tool, the Hopf-Rinow theorem is not available.

\section{BIBLIOGRAPHY}

1. W. Ambrose, Parallel translation of Riemannian curvature, Ann. of Math. vol. 64 (1956) pp. 337-363.

2. E. Cartan, Géometrie des espaces de Riemann, Paris, 1946.

3. N. Hicks, $A$ theorem on affine connections, Illinois J. Math. vol. 3 (1959) pp. 242-254.

HARVARD UNIVERSITY AND

MassachusetTs Institute of Technology 